\title{
Signal averaging for noise reduction in anesthesia monitoring and control with communication channels
}

\author{
Zhi-Bin Tan ${ }^{1}$, Le-Yi Wang ${ }^{2 *}$, Hong Wang ${ }^{3 * *}$ \\ ${ }^{1,2}$ Department of Electrical and Computer Engineering, Wayne State University, Detroit, USA; ${ }^{3}$ Department of Anesthesiology, \\ Wayne State University, Detroit, USA. \\ Email: ${ }^{1}$ au6063@wayne.edu; ${ }^{2}$ lywang@wayne.edu; ${ }^{3}$ howang@med.wayne.edu
}

Received 29 May 2009; revised 14 July 2009; accepted 14 July 2009.

\begin{abstract}
This paper investigates impact of noise and signal averaging on patient control in anesthesia applications, especially in networked control system settings such as wireless connected systems, sensor networks, local area networks, or tele-medicine over a wide area network. Such systems involve communication channels which introduce noises due to quantization, channel noises, and have limited communication bandwidth resources. Usually signal averaging can be used effectively in reducing noise effects when remote monitoring and diagnosis are involved. However, when feedback is intended, we show that signal averaging will lose its utility substantially. To explain this phenomenon, we analyze stability margins under signal averaging and derive some optimal strategies for selecting window sizes. A typical case of anesthesia depth control problems is used in this development.
\end{abstract}

Keywords: Anesthesia Depth; Anesthesia Monitoring; Anesthesia Control; Signal Averaging; Noise Reduction; Open and Closed Loop Systems; Communications; Networked Systems

\section{INTRODUCTION}

To maintain an adequate depth of anesthesia without compromising patient's health, an anesthesiologist usually works as a multi-task feedback controller to roughly regulate the drugs titration while observing a variety of patient outcomes. Automatic anesthesia controller design aims to automatically regulate anesthesia levels by taking account on several physiological measurements and then frees up anesthesiologists for more important tasks

\footnotetext{
*Research of this author was supported in part by the National Science Foundation under ECS-0329597 and DMS-0624849, and Michigan Economic Development Council.

${ }^{* *}$ Research of this author was supported in part by Michigan Economic Development Council and Wayne State University's Research Enhancement Program.
}

in operation. Closed-loop control of anesthesia has been a goal of many researchers since the middle of 20th century. With the emergence of BIS monitor in late 1990s, the interests in closed-loop control of depth of hypnosis is renewed, the most notable works are seen in $[1,2,3,4]$. In an operation room, a wide range of medical devices are connected together or connected to patient through cables for measuring, monitoring and diagnosis. The cable clutter interferes with patient care, creats hazards for clinical staff and delays transport and positioning. To improve the clinical room efficiency and safty, it has been suggested to replace those cables with wireless connections [5].

While anesthesia patient vital signs such as anesthesia depth index, blood pressure, heart rate etc. are transmitted through a noisy wireless channel in a wide area, those transmitted signals will be corrupted by the transmission noise. It is well understood that within most algorithms that reduce effects of random noises on signals and systems, some types of signal averaging are used [6,7]. This is mainly because the laws of large numbers and central limit theorems provide a foundation for noise reduction. The rationale is that when averaging is applied, noises diminish in an appropriate sense. This fundamental understanding leads to algorithms in filtering, signal reconstruction, state estimation, parameter estimation, system identification, and stochastic control. The signal averaging can be used effectively when remote monitoring and diagnosis are involved. On the other hand, signal averaging introduces dynamic delays. Such delays will have detrimental effects on closed-loop systems, even destabilizing the system. Consequently, signal averaging encounters a fundamental performance limitation in feedback systems. To explain this phenomenon, we analyze stability margins under signal averaging and derive some optimal strategies for selecting window sizes. A typical case of anesthesia depth control problems is used in this development.

This paper is organized as follows. Section 2 discusses patient modeling and control in anesthesia appli- 
cations. A typical case is presented with a detailed patient model derived from clinical data. A feedback control is designed to achieve closed-loop system stability, on the basis of state observers and pole placement control. Signal averaging and its effectiveness on open-loop and closed-loop applications are demonstrated in Section 3. We show that while extending filter windows can improve noise attenuation in open-loop systems, it can de-stabilize a closed-loop system, implying a fundamental performance limitation. The idea of using fast sampling is discussed.

Theoretical foundation of our performance analysis is presented in Section 4. It is shown that this can be transformed into a calculation of gain margin of a modified system. Performance limitation is analyzed that leads to an optimal selection of filter windows. It is shown that for a given sampling rate, even optimally designed averaging filters can only have very limited benefits in reducing noise effects. It is shown that noise reduction ratio is proportional to the sampling interval, providing a means of obtaining noise reduction with communication resources. These findings are applied to anesthesia control problems in Section 5. Finally, Section 6 summarizes some issues that are related but not resolved in this paper.

\section{PATIENT MODELS AND FEEDBACK CONTROL}

Real-time anesthesia decisions are exemplified by general anesthesia for attaining an adequate anesthetic depth (consciousness level of a patient), ventilation control, etc $[3,4,8,9]$. One of the most critical requirements in this decision process is to predict the impact of the inputs (drug infusion rates, fluid flow rates, ventilator mode, etc.) on the outcomes (consciousness levels, blood pressures, heart rates, airway pressures, and oxygen saturation, etc.). This prediction capability can be used for control, display, warning, predictive diagnosis, decision analysis, outcome comparison, etc.

\subsection{Patient Models}

The core function of this prediction capability is embedded in establishing a reliable model that relates the drug or procedure inputs to the outcomes in real-time and in individual patients. Due to significant deviations in physical conditions, ages, metabolism, pre-existing medical conditions, and surgical procedures, patient dynamics demonstrate nonlinearity and large variations in their responses to drug infusion. A basic informationoriented model structure for patient responses to drug infusion was introduced in $[10,11,12]$. Propofol (a common anesthesia drug) titration is administered by an infusion pump. The patient's anesthesia depth is measured by a BIS (Bi-Spectrum) monitor $[13,14]$. The monitor provides continuously an index in the range of $[0,100]$ such that the lower the index value, the deeper the anesthesia state. Hence, an index value 0 will indicate "brain dead" and 100 will be "awake".

To establish patient models for monitoring and control, clinical data were collected. One of these data sets is used in this paper. The anesthesia process lasted about 76 minutes, starting from the initial drug administration and continuing until last dose of administration. Propofol was used in both titration and bolus. Fentanal was injected in small bolus amount three times, two at the initial surgical preparation and one near incision. Analysis shows that the impact of Fentanal on the BIS values is minimal. As a result, it is treated as a disturbance and not explicitly modeled in this example. The drug infusion was controlled manually by an experienced anesthesiologist. The trajectories of titration (in $\mu g / \mathrm{sec}$ ) and bolus injection (converted to $\mu \mathrm{g} / \mathrm{sec}$ ) during the entire surgical procedure were recorded, which are shown together with the corresponding BIS values in Figure 1.

The patient was given bolus injection twice to induce anesthesia, first at $t=3$ minute with $20 \mathrm{mg}$ and then at $t=5$ minute with $20 \mathrm{mg}$. They are shown in the figure as $10000 \mu \mathrm{g} / \mathrm{sec}$ for two seconds, to be consistent with the titration units. The surgical procedures were manually recorded. Three major types of stimulation were identified: 1) During the initial drug administration (the first 6 minutes), due to set-up stimulation and patient nervousness. 2) Incision at $t=45$ minute for about 5 minutes duration. 3) Closing near the end of the surgery at $t=60$ minute.

The data from the first 30 minutes are used to determine model parameters and functional forms. For estimating the parameters in the patient block, the data in the interval where the bolus and stimulation impact is minimal (between $t=10$ to $t=30$ minutes) are used. The patient model parameters were identified through Least-Squares estimation method [15].

Under a sampling interval $T=1$ second, which is the standard data transfer interval for the BIS monitor, the combined linear dynamics was estimated. The patient model with propofal infusion rate as the input and BIS measurement as the output was identified as

$$
\begin{aligned}
& P(z) \\
& =\frac{0.01872 z^{2}-0.08813 z+0.09016}{z^{5}-1.159 z^{4}+0.7501 z^{3}-0.5989 z^{2}+0.2984 z-0.2678}
\end{aligned}
$$

The actual BIS response is then compared to the model response over the entire surgical procedure. Comparison results are demonstrated in Figure 2. Here, the inputs of titration and bolus are the recorded real-time data. The model output represents the patient response very well. In particular, the model captures the 

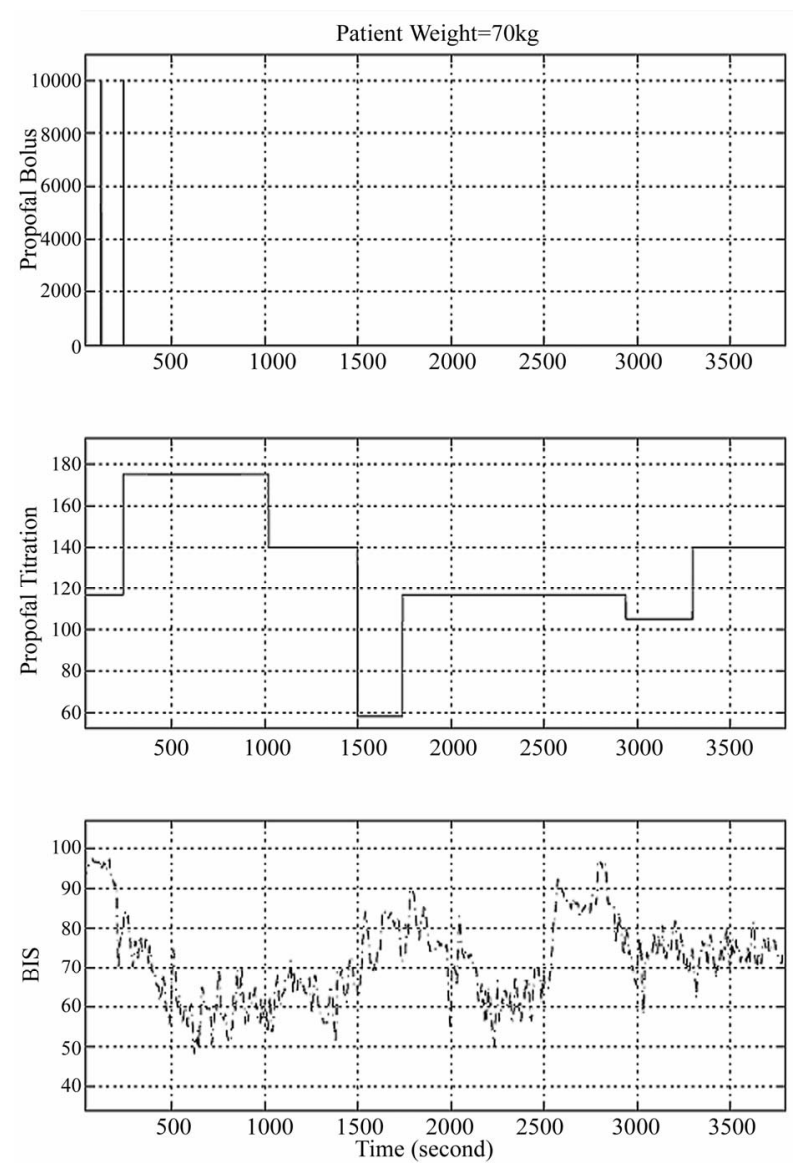

Figure 1. Actual patient responses.

key trends and magnitudes of the BIS variations in the surgical procedure. This indicates that the model structure contains sufficient freedom in representing the main features of the patient response.

\subsection{Feedback Control}

Usually to eliminate steady-state error in tracking
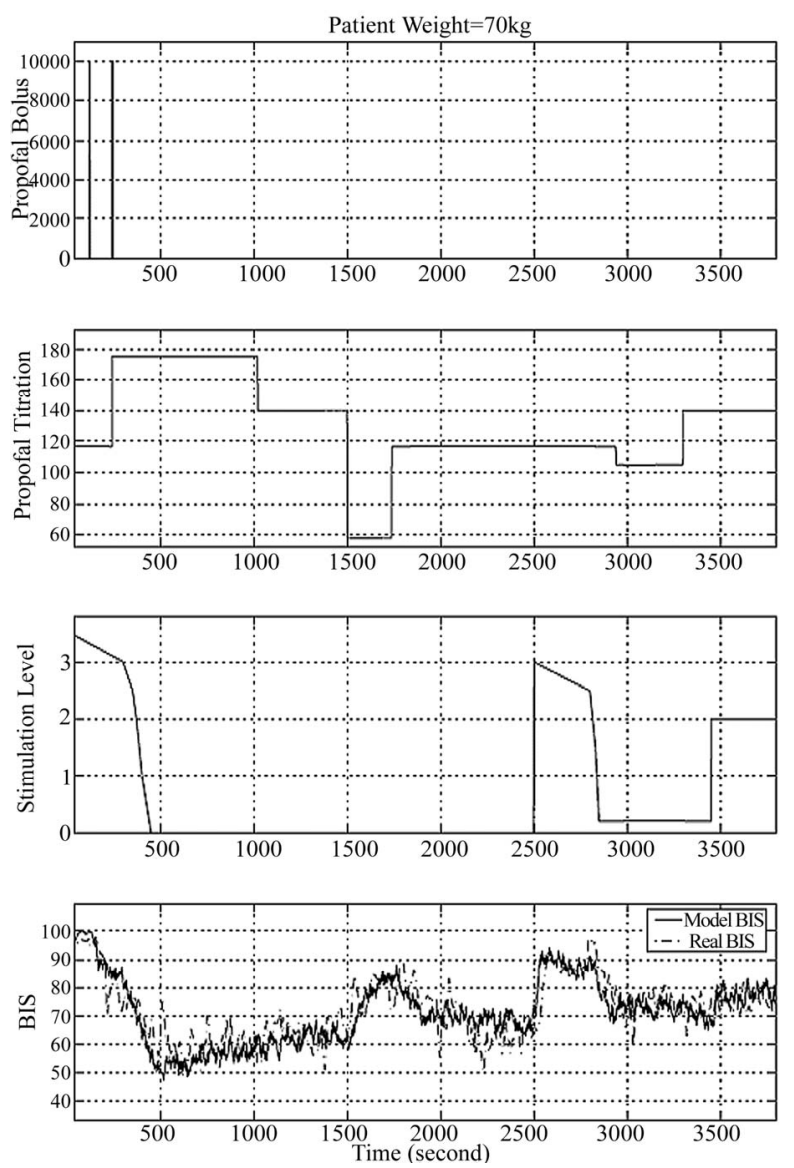

Figure 2. Patient model responses.

control, an integrator is inserted into the system

$$
C(z)=\frac{1}{z-1}
$$

A stabilizing feedback controller is then designed for the patient model (1) by using a full-order observer and pole placement design, leading to

$$
F(z)=\frac{1.234 z^{5}+0.6298 z^{4}-3.644 z^{3}+3.67 z^{2}-1.981 z+0.2479}{z^{6}-2.341 z^{5}+2.284 z^{4}-0.7252 z^{3}-0.4057 z^{2}+0.5714 z-0.08343}
$$

These system components result in a combined openloop system

$$
G(z)=F(z) C(z) P(z)
$$

\section{SIGNAL AVERAGING AND CONTROL PERFORMANCE}

We will use a typical anesthesia control problem to understand impact of communication channels and utility of signal averaging on anesthesia monitoring and control. There are different window functions for signal averaging, such as uniform windows, exponential windows, etc.
They are different only in their forms, but most conclusions for system analysis or error bounds are usually valid for all window types. As a result, we shall use the exponential windows to carry out our analysis. A signal averaging by exponential decaying weighting of rate $0<\alpha<1$ is

$$
h_{k}=(1-\alpha) \sum_{i=-\infty}^{k} \alpha^{k-i} x_{i}
$$

whose transfer function is

$$
F_{\alpha}(z)=\frac{(1-\alpha) z}{z-\alpha}
$$




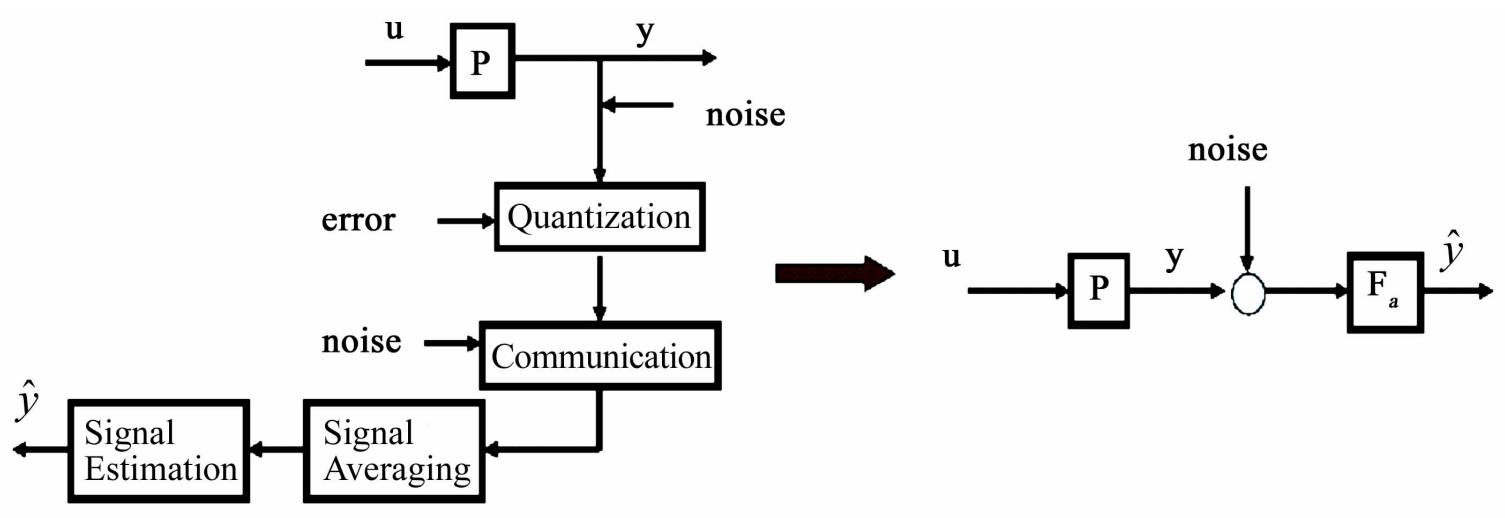

Figure 3. Signal filtering in open-loop system.

\subsection{Open-Loop Systems}

In wireless-based monitoring and diagnosis applications, the system is running in open-loop. In this case quantization errors and communication noises can be grouped as an additive noise to the patient output $y$. When signal averaging is applied to reduce noise effects, the resulting system can be represented by the block diagram in Figure 3.

Figure 4 illustrates impact of filtering on open loop systems. In open loop applications, filtering will not de-stabilize the system. Consequently, one may choose a window of long horizon to reduce the effects of noise. It is apparent that the longer the averaging window, the less the noise effect on the signal. However, it is also observed that signal averaging slows down system's
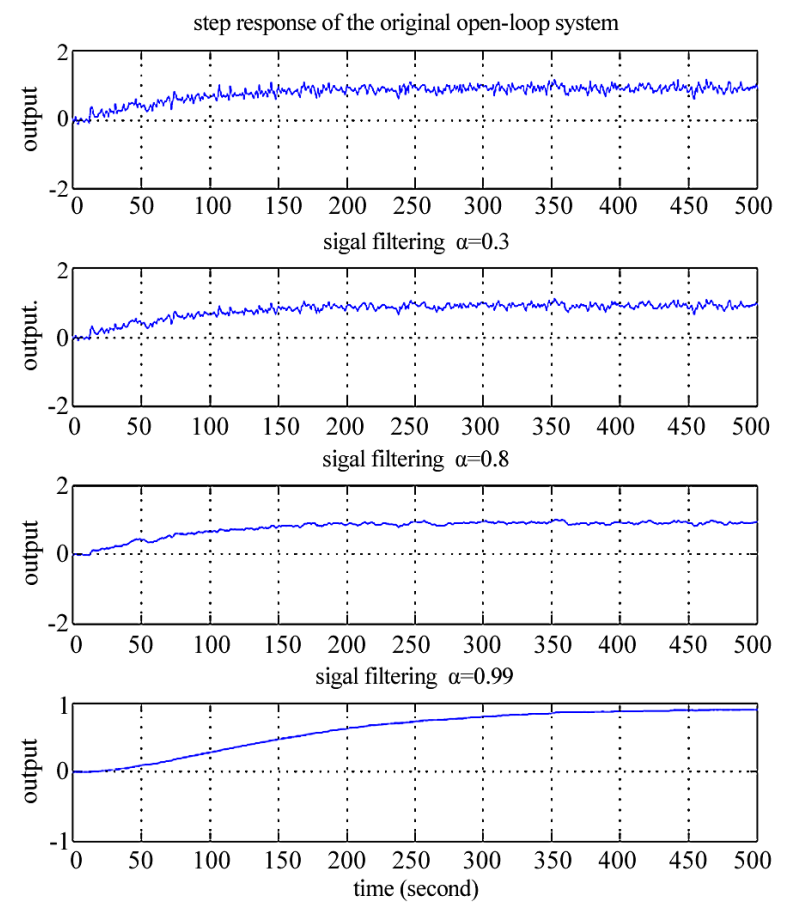

Figure 4. Effects of signal averaging on open-loop systems. response to the input. In other words, filtering introduces a dynamic delay. This delay has very important implication in the closed-loop applications.

\subsection{Closed-Loop Systems}

On the other hand, if feedback control for anesthesia management decisions is intended, signal filtering becomes part of a closed-loop system. When signal averaging is applied, the averaging filter $\boldsymbol{F}_{a}$ is inserted into the system, resulting in a modified closed-loop system shown in Figure 5.

The close-loop system equations are:

$$
y_{k}=G e_{k}, \quad e_{k}=r_{k}-F_{\alpha}\left(y_{k}+d_{k}\right)
$$

Then

$$
y_{k}=G r_{k}-G F_{\alpha} y_{k}-G F_{\alpha} d_{k}
$$

and

$$
y_{k}=H_{r} r_{k}+H_{\alpha} d_{k}
$$

where,

$$
H_{r}=\frac{G}{1+F_{\alpha} G} ; \quad H_{\alpha}=\frac{-F_{\alpha} G}{1+F_{\alpha} G}
$$

Figure 6 illustrates impact of filtering on closed loop systems. Although signal filtering can reduce the noise effect of the signal, it introduces a dynamic delay which has detrimental effects on the closed-loop system. The plots confirm that when filtering window is long the filter can destabilize the closed-loop system. Even when the filtering window size is small, its effectiveness is not very obvious. This example suggests that in closed-loop applications signal filtering has limited effectiveness. This understanding will be used to introduce new methods to reduce noise effects in such applications.

\subsection{Re-Sampling}

The plant in this case is identified as a 5 th order difference equation in (1). The system can be well approxi- 


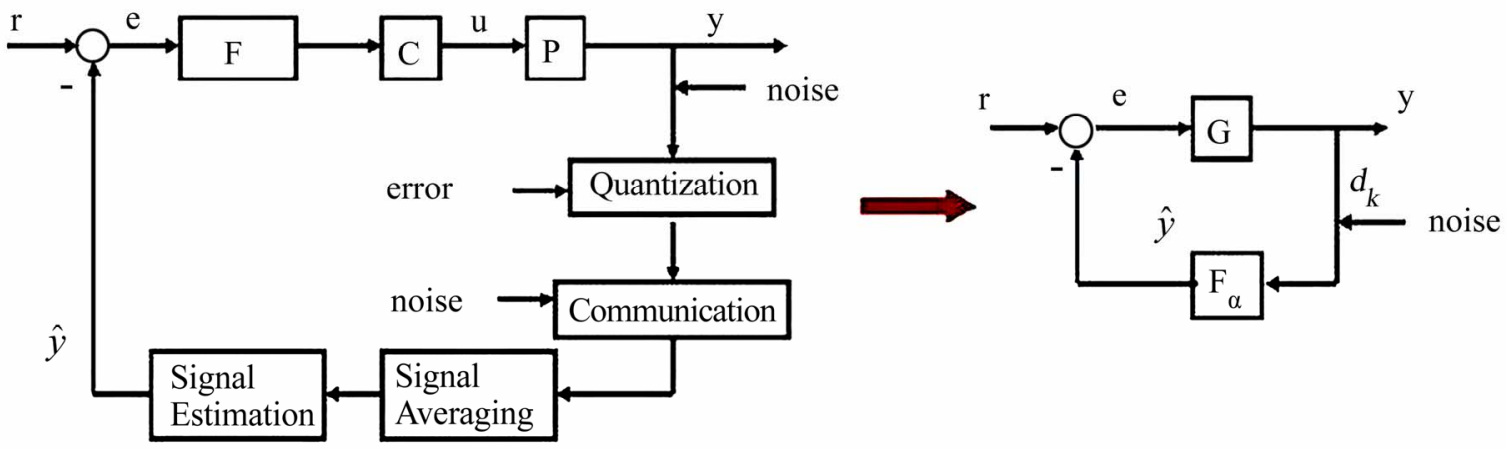

Figure 5. System modules and their equivalent representation.
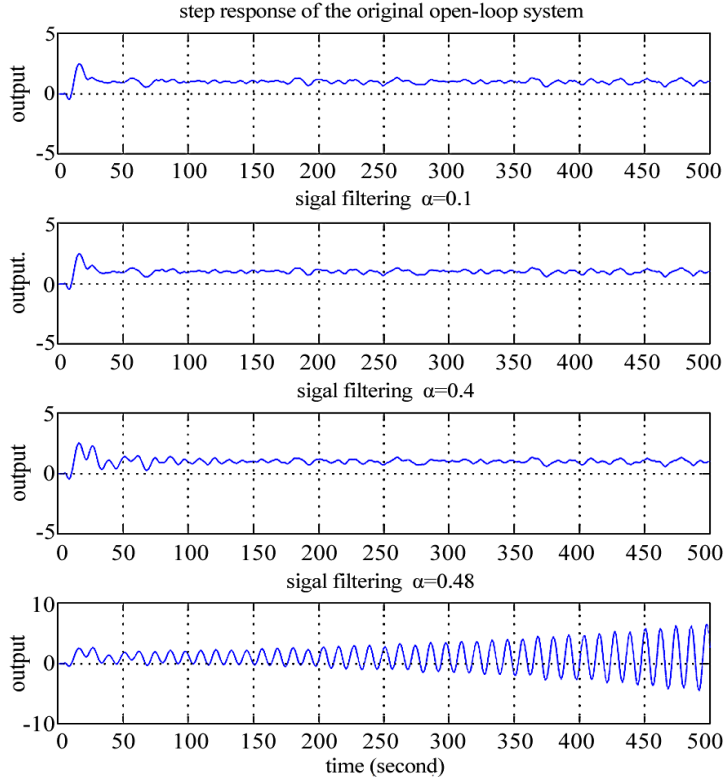

Figure 6. Effects of signal averaging on closed-loop systems.

mated by a continuous-time system that consists of a pure time delay and a first-order dynamics, sampled with sampling interval $T=1$ second. Let a continuous-time system be

$$
P(s)=e^{-5 s} \frac{0.93}{73 s+1}
$$

The step responses of the original system (1) and the simplified system $P(\mathrm{~s})$ are shown in Figure 7.

This approximation allows us to use smaller sampling intervals to re-sample the output of the system. The benefits of re-sampling will become clear after some theoretical analysis in the next section.

\section{ANALYSIS OF STABILITY AND PERFORMANCE}

Definition 1 The stability margin against exponential averaging, abbreviated as $\alpha$-margin and denoted by $\alpha_{\max }(G)$, is the largest $0 \leq \alpha \leq 1$ such that for all

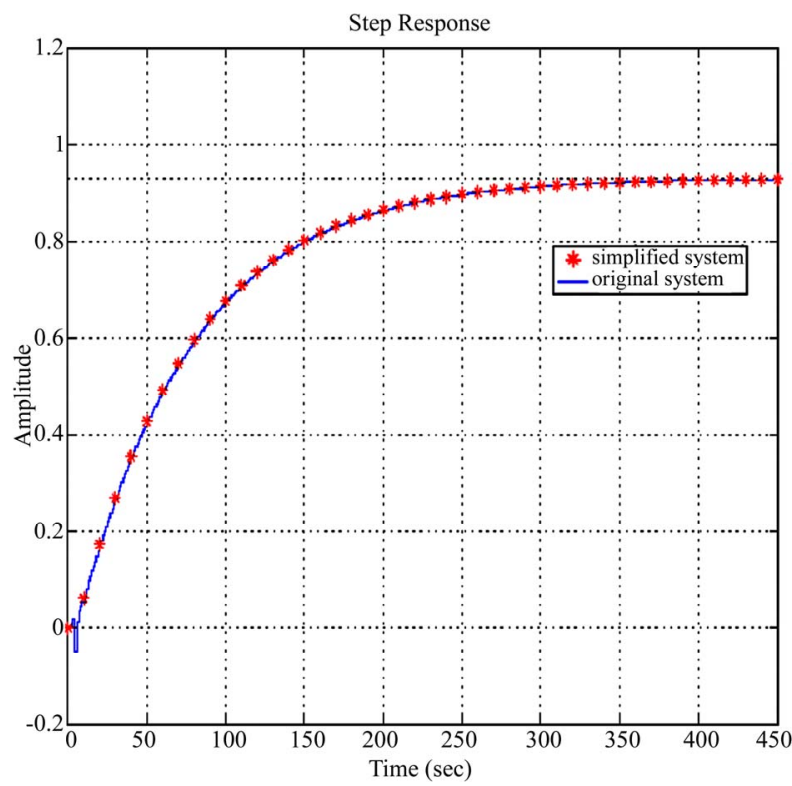

Figure 7. Step responses of the original system and the simplified system.

$0 \leq \alpha<\alpha_{\max }(G)$, the close-loop system (6) is stable and the system is unstable if $\alpha>\alpha_{\max }(G)$. If the close-loop system is stable for all $\alpha$, we denote $\alpha_{\max }(G)=1$.

Suppose that the input to the filter is a noise corrupted constant

$$
x_{k}=\theta+d_{k}
$$

An exponential window of rate $0<\alpha<1$ is applied to this signal and its output is

$$
h_{k}=(1-\alpha) \sum_{i=-\infty}^{k} \alpha^{k-i} x_{i}=\theta+(1-\alpha) \sum_{i=-\infty}^{k} \alpha^{k-i} d_{i}=\theta+\varepsilon_{k}
$$

where

$$
\varepsilon_{k}=(1-\alpha) \sum_{i=-\infty}^{k} \alpha^{k-i} d_{i}
$$

If $d_{i}$ is i.i.d. (independent and identically distributed) 
with $E d_{i}=0$ and $E d_{i}^{2}=\sigma^{2}$, then $E \varepsilon_{k}=0$ and

$$
E \varepsilon_{k}^{2}=\frac{1-\alpha}{1+\alpha} \sigma^{2}
$$

Consequently, using $h_{k}$ as an estimate of $\theta$ can reduce errors by $1-\alpha 1+\alpha$. We will call $\alpha$ as the decaying rate. Convergence (in the mean square sense) is achieved when $\alpha \rightarrow 1: \lim _{\alpha \rightarrow 1} E \varepsilon_{k}^{2}=0$.

Consider an exponential filter

$$
F(s)=\frac{1}{\lambda s+1}
$$

whose impulse response is

$$
f(t)=\frac{1}{\lambda} e^{-t / \lambda}, t \geq 0
$$

Note that

$$
\int_{0}^{\infty} f(t) d t=1
$$

Now, the input-output relationship of this filter is

$$
\begin{aligned}
y(t) & =\int_{-\infty}^{t} f(t-\tau) x(\tau) d \tau \\
= & \frac{1}{\lambda} \int_{-\infty}^{t} e^{-(t-\tau) / \lambda} x(\tau) d \tau
\end{aligned}
$$

When the signals are sampled with sampling interval $\boldsymbol{T}$, we denote $x_{k}=x(k T)$ and $y_{k}=y(k T)$. If $\alpha$ is related to $\lambda$ and $\boldsymbol{T}$ by $\alpha=e^{-T / \lambda}$, we have

$$
\lim _{T \rightarrow 0} \frac{T}{\lambda(1-\alpha)}=1
$$

For small $\boldsymbol{T}, y(t)$ is approximated by

$$
\begin{aligned}
& y_{k}=y(k T)=\frac{1}{\lambda} \int_{-\infty}^{t} e^{-(t-\tau) / \lambda} x(\tau) d \tau \\
& \approx \frac{T}{\lambda} \sum_{i=-\infty}^{k}\left(e^{-T / \lambda}\right)^{k-i} x_{i}=\frac{T}{\lambda(1-\alpha)}(1-\alpha) \sum_{i=-\infty}^{k} \alpha^{k-i} x_{i} \approx(1-\alpha) \sum_{i=-\infty}^{k} \alpha^{k-i} x_{i}
\end{aligned}
$$

In other words, for system analysis, we may approximate the discrete-time filter in (3) by its continuous-time counterpart in (9). These relationships between discretetime averaging and continuous time averaging will be used to derive stability margins.

\subsection{Stability Margin against Exponential Averaging}

The relationship between $\alpha$ and $\lambda$ will allow us to focus on stability analysis in continuous time systems and then transform the results to the discrete-time filters.
This is stated in the following theorem.

Theorem 1 If the exponential stability margin in the continuous-time domain is $\lambda_{\max }$, then

$$
\lim _{T \rightarrow 0} \frac{T}{-\ln \alpha_{\max }}=\lambda_{\max }
$$

Proof: This follows from the relationship

$$
\alpha=e^{-T / \lambda}
$$

We now concentrate on calculation of $\lambda_{\max }$.

Definition 2 The stability margin against exponential averaging for the continuous-time closed-loop system, abbreviated as continuous exponential A-margin and denoted by $\lambda_{\max }(G)$, is the smallest $\lambda>0$ under which the closed-loop system becomes unstable. If the closed-loop system remains stable for all $\lambda>0$, we denote $\lambda_{\max }(G)=\infty$.

Suppose $G(s)=N(s) / D(s)$ where $n(s)$ and $d(s)$ are polynomial functions of $s$ and coprime (that is, $N(s)$ and $D(s)$ do not have common zeros). Then $\lambda_{\max }$ is the largest $\lambda>0$ before the closed-loop system becomes unstable. Consider the characteristic equation of the closed-loop system

$$
1+F_{\lambda}(s) G(s)=1+\frac{1}{\lambda s+1} \frac{N(s)}{D(s)}=0
$$

or

$$
\lambda s D(s)+D(s)+N(s)=0
$$

which leads to

$$
1+\lambda \frac{s D(s)}{D(s)+N(s)}=0
$$

This expression leads to the following conclusion.

Theorem 2 The exponential A-margin $\lambda_{\max }(G)$ of $G(s)$ is the gain margin of

$$
H(s)=\frac{s D(s)}{D(s)+N(s)}
$$

We make several interesting observations from (12). First, from (11), $\lambda_{\max }$ may be calculated by using the Routh-Hurwitz test. Second, (12) is in a standard form for using root locus technique. So, we may plot the root locus of the system (13) (it is an improper system) and detect the $\lambda$ value that reaches marginal stability, which will be $\lambda_{\max }$. The root locus plot starts at the poles of system (13) which are precisely the poles of the closed-loop system without the averaging filter. Since 


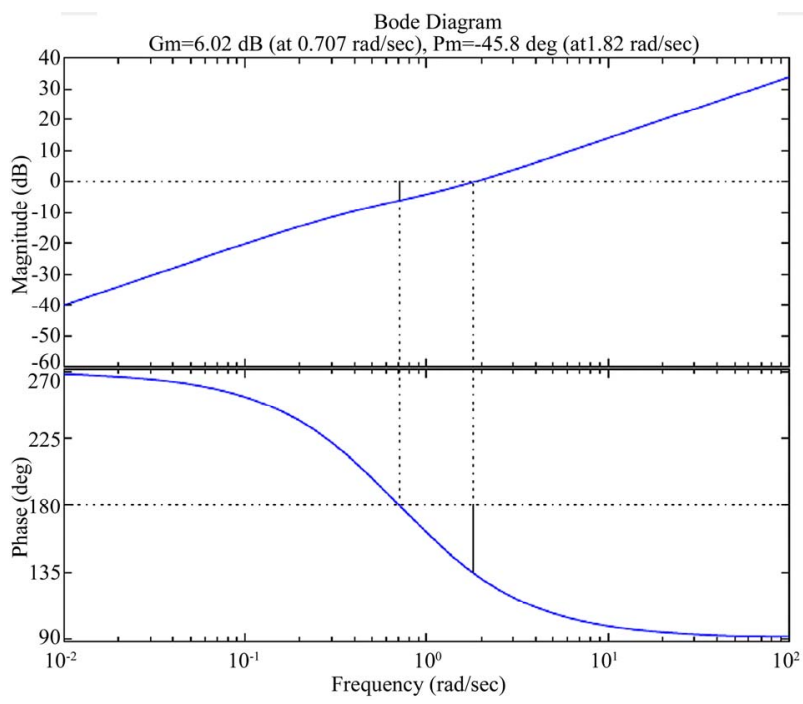

Figure 8. Using bode plots to obtain the gain margin.

the closed-loop system is stable, for small $\lambda$ the closedloop system with the filter will remain stable. The root locus plot moves towards the zeros of system (13) which are the poles of the open-loop system. Hence, if the open-loop system is unstable, the exponential A-margin is always finite.

Example 1 Suppose $G(s)=(s+2) /(s-1)$. Then,

$$
H(s)=\frac{s D(s)}{D(s)+N(s)}=s^{2}-s 2 s+1
$$

The gain margin can be obtained by using the Matlab function "margin" (which gives $\lambda_{\max }=2$ ) or by plotting the bode plot as shown in Figure 1 which gives $\lambda_{\max }=6.02 \mathrm{~d} B=2$. Alternatively, from

$$
(2 s+1)+\lambda\left(s^{2}-s\right)=\lambda s^{2}+(2-\lambda) s+1=0
$$

we can calculate $\lambda_{\max }=2$ by the Routh-Hurwitz method.

\subsection{Performance Analysis}

Within the A-margin, what is the benefit of signal averaging? On one hand, signal averaging can reduce noise effect. On the other hand, averaging introduces delays and reduces closed-loop system performance. Consequently, an optimal choice of averaging becomes an issue.

Similarly, the continuous time close-loop system equations are:

$$
y=\frac{G}{1+F_{\lambda} G} r-\frac{F_{\lambda} G}{1+F_{\lambda} G} d
$$

Here, we denote

$$
H_{\lambda}=\frac{-F_{\lambda} G}{1+F_{\lambda} G}
$$

If $d$ is a white noise, noise attenuation aims to reduce the $L^{2}$ norm of $H_{\lambda}$. Naturally, for optimal noise reduction, we should select

$$
\eta=\inf _{0<\lambda<\lambda_{\max }}\left\|H_{\lambda}\right\|_{2}
$$

Example 2 For the system in Example 1, when $\lambda$ takes values $0,0.1, \ldots 0.9$, the corresponding $H^{2}$ norms for the closed-loop system $H_{\lambda}$ are

$\begin{array}{lllllllllll}\lambda & 0 & 0.1 & 0.2 & 0.3 & 0.4 & 0.5 & 0.6 & 0.7 & 0.8 & 0.9\end{array}$

$\begin{array}{lllllllllll}\eta & 6.22 & 7.00 & 7.87 & 9.00 & 10.49 & 12.59 & 15.73 & 20.96 & 31.44 & 62.85\end{array}$

The monotone increase of the $L^{2}$ norms indicates that for this system, averaging cannot reduce noise impact on the output. As a result, there should be no averaging for this system.

Example 3 For another example, consider a system

$$
G(s)=\frac{s^{2}+2 s-1}{s^{2}-s+4}
$$

The closed-loop system's characteristic equation is

$$
\begin{aligned}
& \lambda s D(s)+D(s)+N(s) \\
& =\lambda s^{3}+(2-\lambda) s^{2}+(1+4 \lambda) s+3=0
\end{aligned}
$$

It can be calculated by the Routh-Hurwitz method that $\lambda_{\max }=1.366$. The $H^{2}$ norm of $H_{\lambda}$ as a function of $\lambda$ is plotted in Figure 3. The optimal averaging occurs at $\lambda=0.59$ with the norm $\mathrm{PH}_{0.59} \mathrm{P}_{2}=2.5263$

From the relationship $\alpha=e^{-T / \lambda}$, for small sampling interval $T$,

$$
\alpha=e^{-T / \lambda}=e^{-T / 0.59}=e^{-1.7 T}
$$

is the optimal rate for averaging in the discrete-time domain. For example, if $T=0.01$, we obtain $\alpha=0.983$.

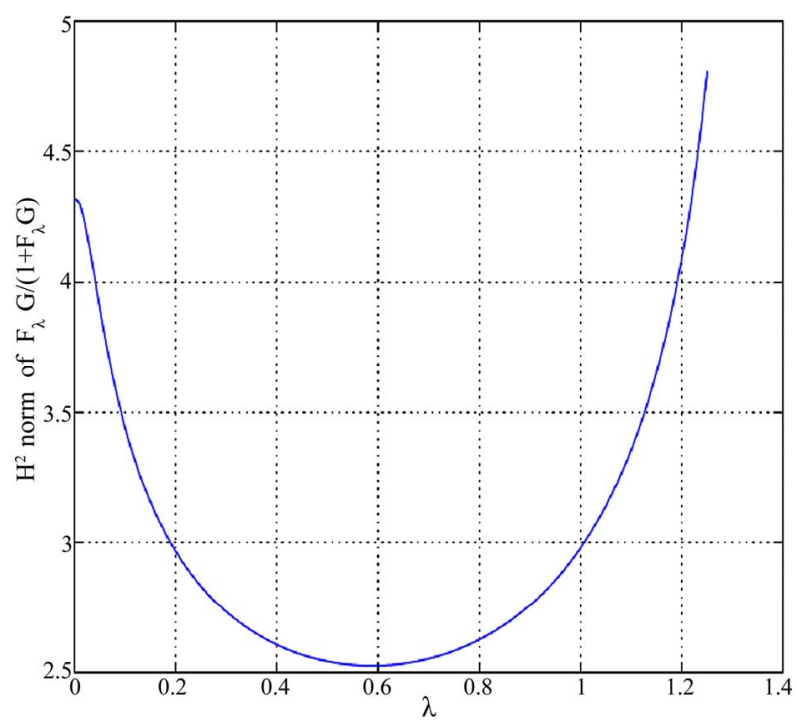

Figure 9. Optimal averaging rate. 


\subsection{Fast Sampling for Disturbance Attenuation}

Although the optimal $L^{2}$ performance $\eta$ in (16) cannot be improved for the continuous-time system, noise attenuation in the sampled system can be further improved.

We first establish a relationship between the $L^{2}$ norm of the continuous-time system and the $l^{2}$ norm of its sampled system. Suppose that the disturbance sequence $d_{k}$ passes through a $\mathrm{ZOH}$ of interval $T$ to become $d(t)$. The continuous-time system $H$ is stable with impulse response $h(t)$. Then,

$$
y(t)=\int_{0}^{t} h(t-\tau) d(\tau) d \tau
$$

Suppose $d_{k}$ is a pulse sequence, $d_{0}=1$, and $d_{k}=0$, $k \neq 0$. Then, $d(t)=1,0 \leq t<T$, and $d(t)=0$, otherwise. Under this input

$$
y(t)=\int_{0}^{T} h(t-\tau) d \tau
$$

Hence, the sampled values of $y(t)$, which form the pulse response of the sampled system, become

$$
y_{k}=y(K T)=\int_{0}^{T} h(k T-\tau) d \tau
$$

For small $T$ this can be approximated by

$$
y_{k}=T h(k T)
$$

We note that for small $T$,

$$
\mathrm{PHP}_{2}^{2}=\int_{0}^{\infty} h^{2}(t) d t \approx T \sum_{k=0}^{\infty} h^{2}(k T)
$$

Consequently, if we use $\tilde{H}$ and $\tilde{h}_{k}$ to denote the sampled system and its impulse response, we have $\tilde{h}_{k}=T h(k T)$ and

From

$$
\mathrm{P} \tilde{H} \mathrm{P}_{2}^{2}=\mathrm{P} \tilde{h}_{k} \mathrm{P}_{2}^{2}=T^{2} \sum_{k=0}^{\infty} h^{2}(k T)=T \mathrm{P} H \mathrm{P}_{2}^{2}
$$

$$
y_{k}=\sum_{i=0}^{k} \widetilde{h}_{k-i} d_{i}
$$

if $d_{k}$ is i.i.d., mean zero and variance $\sigma^{2}$, then

$$
\begin{aligned}
& \sigma_{k}^{2}=E y_{k}^{2}=\sum_{i=0}^{k} \sum_{j=0}^{k} \tilde{h}_{k-i} E d_{i} d_{j} \tilde{h}_{k-j} \\
& =\sigma^{2} \sum_{i=0}^{k} \tilde{h}_{k-i}^{2} \leq \sigma^{2} \mathrm{P} \tilde{h}_{k} \mathrm{P}_{2}^{2}=\sigma^{2} T \mathrm{PH} \mathrm{P}_{2}^{2}
\end{aligned}
$$

In fact,

$$
\sigma_{\max }^{2}=\sup _{k} \sigma_{k}^{2} \approx \sigma^{2} T \mathrm{P} H \mathrm{P}_{2}^{2}
$$

If $\mathrm{PHP}_{2}^{2}$ is optimized, then $\mathrm{PHP}_{2}^{2}=\eta^{2}$ as in (16). Consequently, the noise reduction ratio can be expressed as

$$
\tilde{\eta}^{2}=T \eta^{2}
$$

This is a relationship between noise reduction in the sampled system and the optimal $L^{2}$ norm of the continuous-time system. This analysis concludes that using faster sampling (smaller $T$ ) can reduce the noise effects.

\section{CONTROL WITH SIGNAL AVERAGING}

Findings from Section 4 provide some useful design guidelines. 1) Signal averaging is beneficial in reducing noise effects. 2) Effectiveness of signal averaging in closed-loop systems varies substantially with the filter windows or decaying rates. There is an optimal decaying rate at which signal filtering becomes most effective. 3) When the filter window is optimally selected, further noise attenuation can only be achieved by increasing the sampling rates. 4) Increasing sampling rates incurs higher bandwidth requirements for communications. When channel bandwidths are limited, there is a fundamental performance limit for noise attenuation. This is a unique feature for closed-loop systems. In open-loop applications, convergence can be obtained by applying signal averaging over a very long horizon. However, this cannot be applied to closed-loop systems since long windows of filtering destabilize the feedback system.

\subsection{Anesthesia Applications}

We now apply these understandings to anesthesia control systems. The open-loop transfer function in (2) can be derived as

$$
G(z)=\frac{N(z)}{D(z)}
$$

with

$$
\begin{aligned}
N(z)= & 0.02311 z^{7}-0.09699 z^{6} \\
& -0.01243 z^{5}+0.4466 z^{4}-0.689 z^{3} \\
& +0.5101 z^{2}-0.2005 z+0.02235
\end{aligned}
$$

and

$$
\begin{aligned}
D(z)= & z^{12}-4.5 z^{11}+9.248 z^{10}-11.48 z^{9} \\
& +9.576 z^{8}-5.684 z^{7}+2.528 z^{6}-0.7518 z^{5} \\
& -0.2721 z^{4}+0.6608 z^{3}-0.507 z^{2} \\
& +0.2003 z-0.02234
\end{aligned}
$$

Since the open loop system is unstable, the stability margin of the closed-loop system with inserted averaging window is always limited. The closed-loop system's stability concerns have already been depicted in Figure 
6. The closed-loop system's $H^{2}$ norm, which defines the system's ability in noise attenuation, is shown in Figure 10.

Then, the closed-loop system's step response is simulated when the filter is optimally selected and shown in Figure 11. The system inherent sampling rate is $T=1$ second.

While re-sampling is performed with $T=1$, the $H^{2}$ norm of the closed-loop system will be reduced further. For reduced sampling intervals, improvements of noise attenuation are illustrated in Figure 12.

\subsection{Discussions}

It can be seen from Figure 10 that the optimal filter decaying rate is $\alpha_{\text {opt }}=0.1300$ with the corresponding $H^{2}$ norm 9.0872 when $T=1$. The closed-loop system is stable and it's step response has much fluctuation in steady state. From the relationship, $\alpha_{\text {opt }}=e^{-T / \lambda_{o p t}}=e^{-1 / \lambda_{o p t}}$, we obtain $\lambda_{\text {opt }}=0.49$. This leads to the optimal choice of

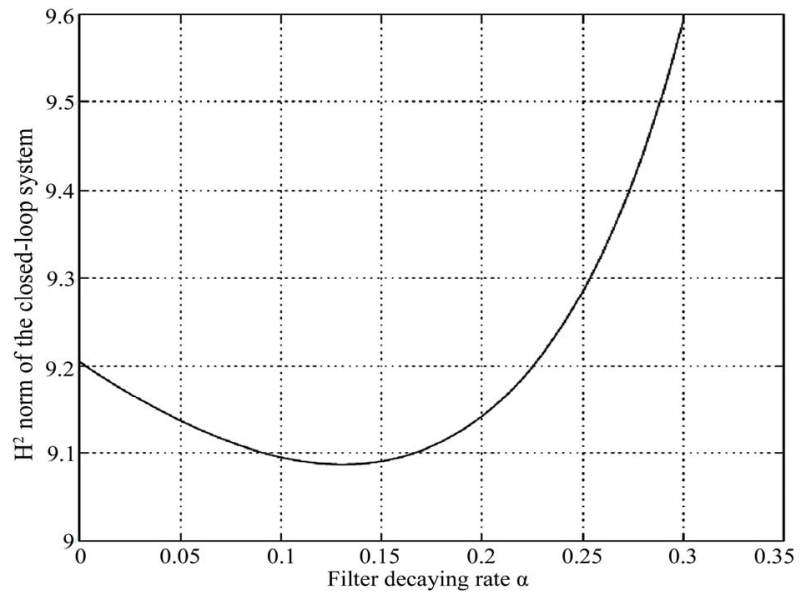

Figure 10. Closed-loop system performance vs. filter decaying rates.

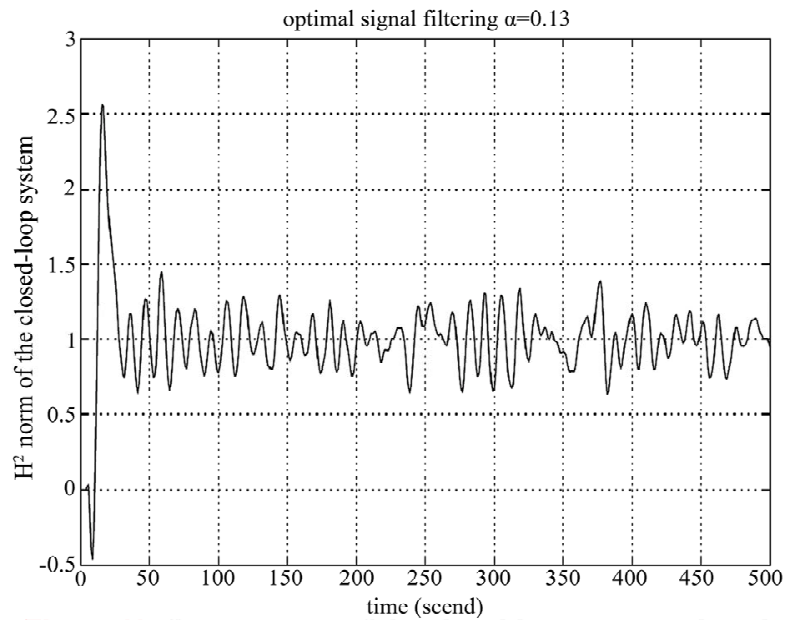

Figure 11. Step response of the closed-loop system when the filter is optimally selected, and sampling interval $T=1$.
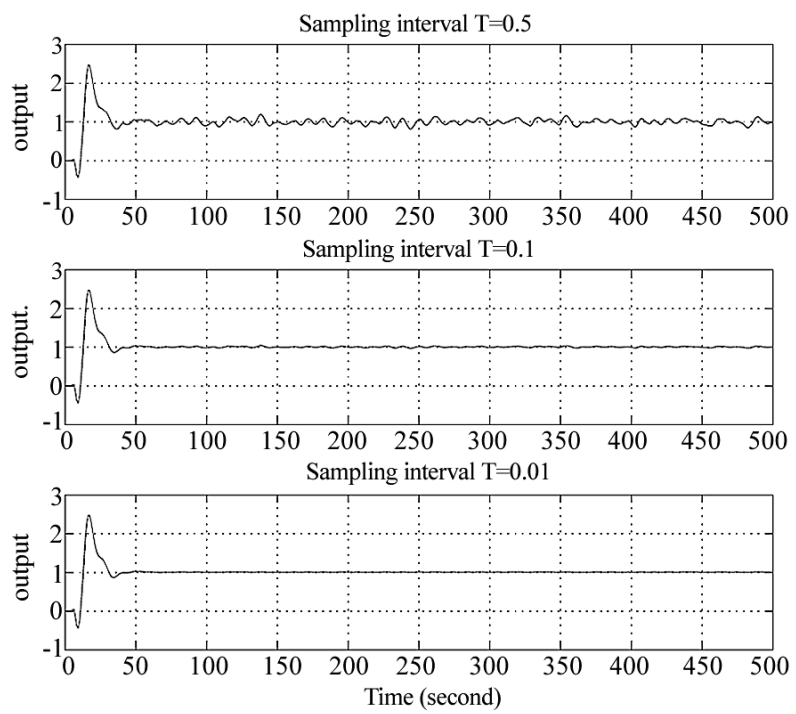

Figure 12. The closed-loop system performance for reduced sampling intervals.

decaying rate when the sampling interval $T$ is reduced from 1 as

$$
\alpha=e^{-T / \lambda_{\text {opt }}}=e^{-T / 0.49}=e^{-2.04 T} .
$$

When sampling rate is increased to $1 T$, the $H^{2}$ norm of the closed-loop system will be reduced to $9.0872 T$ as established in (17). Figure 12 illustrates the step responses of the closed-loop system with sampling interval $T=0.5, T=0.1$ and $T=0.01$ second respectively. The steady state fluctuation of the step response is decreasing with the reduced sampling intervals.

\section{CONCLUSIONS}

The impact of communication channels on feedback control in anesthesia applications in wireless based systems was investigated in this paper. Such systems involve communication channels which are corrupted by noises and have limited bandwidth resources. Signal averaging is the fundamental method in dealing with stochastic noises and errors. It is used effectively in reducing noise effects when only remote monitoring and diagnosis are involved. However, the case is different when feedback is intended.

Our results show that the decaying rate of the averaging window has significant impact on the performance of the close-loop system. When $\alpha$ is larger than some value, the close-loop system becomes unstable. A concept of stability margins against exponential averaging is introduced. Its calculation can be performed by either the Routh-Hurwitz method or the root-locus method on a modified system. Furthermore, the strategy for choosing the optimal decaying rate is derived. Our results conclude that fast sampling must be used for improving noise reduction after optimal filter design. The analysis and design method is applied to anesthesia patient con- 
trol problems.

Our analysis is conducted on the basis of the linear systems. Actually, anesthesia patient models contain nonlinearity. Our future work will consider analysis of nonlinear systems.

\section{REFERENCES}

[1] Nunes, C. S., Mendonca, T., Lemos, J. M., and Amorim, P., (2007) Predictive adaptive control of the bispectral index of the EEG (BIS): Exploring electromyography as an accessible disturbance, Mediterranean Conference on Control and Automation, Athens-Greece.

[2] Gentilini, A., Rossoni-Gerosa, M., and Morari, M., et al, (2001) Modeling and closed-loop control of hypnosis by means of bispectral index (BIS) with isoflurane, IEEE Trans. on Biomedical Engineering, 48, 874-889,.

[3] Dong, C., Kehoe, J., Henry, J., Ifeachor, E. C., Reeve, C. D., and Sneyd, J. R., (1999) Closed-loop computer controlled sedation with propofol, Proc. of the Anaesthetic Research Society, 631.

[4] Zhang, X. S., Roy, R. J., and Huang, J. W., (1998) Closedloop system for total intravenous anesthesia by simultaneously administering two anesthetic drugs, Proc. of the 20th Annual International Conference of the IEEE Engineering in Medicine and Biology, 3052-3055.

[5] Goldman, J. M., (2006) Medical device connectivity for improving safety and efficiency, American Society of Anesthesiologists Newsletters, 70(5),

http://www.asahq.org/Newsletters/2006/05-06/goldman0 5 06.html.
[6] Talbot, S. L. and Boroujeny, B. F., (2008) Spectral method of blind carrier tracking for OFDM, IEEE Transactions on Signal Processing, 56(7).

[7] Bataillou, E., Thierry, E., Rix, H., and Meste, O., (1995) Weighted averaging using adaptive estimation of the weights, Signal Processing, 44, 51-66.

[8] Eisenach, J. C., (1999) Reports of Scientific MeetingsWorkshop on Safe Feedback Control of Anesthetic Drug Delivery, Anesthesilogy, 91, 600-601.

[9] Linkens, D. A., (1992) Adaptive and intelligent control in anesthesia, IEEE Control Systems Magazine, 6-11.

[10] Wang, L. Y. and Wang, H., (2002) Control-oriented modeling of BIS-based patient response to anesthesia infusion, Internat. Conf. Math. Eng. Techniques in Medicine and Bio. Sci., Las Vegas.

[11] Wang, L. Y. and Wang, H., (2002) Feedback and predictive control of anesthesia infusion using control-oriented patient models, Internat. Conf. Math. Eng. Techniques in Medicine and Bio. Sci., Las Vegas.

[12] Wang, L. Y., Wang, H., and Yin, G., (2002) Anesthesia infusion models: Knowledge-based real-time identification via stochastic approximation, 41st IEEE Cont. and Dec. Conf., Las Vegas.

[13] Gan, T. J., et al., (1997) Bispectral index monitoring allows faster emergence and improved recovery from propofol, Alfentanil, and Nitrous Oxide Anesthesia, Anesthesiology, 87, 808-815.

[14] Rosow, C. and Manberg, P. J., (1998) Bispectral index monitoring, Annual of Anesthetic Pharmacology, 2, 1084 2098.

[15] Ljung, L. and Söderström, T., (1983) Theory and Practice of Recursive Identification, MIT Press, Cambridge, MA. 